\title{
Effect of Halo Neutrals on Fast-Ion Charge Exchange Spectroscopy Measurements in LHD
}

\author{
Takafumi ITO, Masaki OSAKABE ${ }^{1)}$, Katsumi IDA ${ }^{1)}$, Mikiro YOSHINUMA ${ }^{1)}$, \\ Masahiko KOBAYASHI ${ }^{1)}$, Sadayoshi MURAKAMI ${ }^{2)}$, Motoshi GOTO $^{1)}$, Yasuhiko TAKEIRI ${ }^{1)}$, \\ Detlev REITER ${ }^{3)}$, Shoichi OKAMURA ${ }^{1)}$ and LHD Experimental Group ${ }^{1)}$ \\ The Graduate University for Advanced Studies, Toki 509-5292, Japan \\ ${ }^{1)}$ National Institute for Fusion Science, Toki 509-5292, Japan \\ ${ }^{2)}$ Department of Nuclear Engineering, Kyoto University, Kyoto 606-8501, Japan \\ ${ }^{3)}$ Institut für Plasmaphysik, Forschungszentrum Jülich GmbH, D-52425 Juelich, Germany
}

(Received 22 December 2009 / Accepted 6 May 2010)

\begin{abstract}
A fast-ion charge exchange spectroscopy (FICXS) diagnostic was recently installed on the Large Helical Device (LHD). The aim of this diagnostic is to evaluate fast-ion confinement property by measuring energyresolved fast-ion spatial profiles in LHD. To accurately evaluate the FICXS profile, the effect of halo neutrals, which are created by the charge exchange between bulk ions and injected neutral beams (NBs), must be treated properly in the current analysis of the FICXS spectra in the LHD. We have estimated the effect of halo neutrals on the measurement from the shapes of Doppler-shifted $\mathrm{H}$-alpha spectra. The ratio of halo neutrals to the injected NBs on the lines of sight (LOSs) is estimated by using the EIRENE-3D Monte Carlo code. The results of these two methods agreed well. This indicates that the effect of halo neutrals should be considered when interpreting the FICXS diagnostic data as well as that from the injected NB, when the probing NB is radially injected and the emitted photons are observed from perpendicular LOSs.
\end{abstract}

(c) 2010 The Japan Society of Plasma Science and Nuclear Fusion Research

Keywords: fast-ion, charge exchange spectroscopy, neutral particle transport

DOI: $10.1585 /$ pfr.5.S2099

\section{Introduction}

The confinement of fast-ions is a common physics concern for toroidal magnetic devices because plasma sustainment by fusion-born alphas is necessary in fusion reactors. Therefore, it is important to evaluate the confinement properties of fast-ions in such devices. In the Large Helical Device (LHD), the effects of magnetic ripples and magnetohydrodynamic (MHD) instabilities on fast-ion confinement have been investigated by fast neutral diagnostics [1-3]. Since the diagnostics observe a limited region in the velocity space of fast ions, it is very difficult to investigate their overall confinement properties.

In the LHD, a fast-ion charge exchange spectroscopy (FICXS) diagnostic was recently applied [4]. This method is similar to the fast-ion deuterium-alpha (FIDA) that was developed on DIII-D [5-7]. These methods can evaluate the spatial and energy distributions of fast ions from the Doppler-shifted Balmer alpha $\left(\mathrm{H}_{\alpha}\right.$ or $\left.\mathrm{D}_{\alpha}\right)$ spectra of charge-exchanged fast neutrals from an injected neutral beam (NB). An advantage of this method is that it can yield global confinement properties of fast ions.

In a FICXS measurement, $\mathrm{H}_{\alpha}$ light from the following three processes must be considered:

$$
\begin{aligned}
& \mathrm{H}_{\text {fast }}^{+}+\mathrm{H}_{\mathrm{NB}}^{0} \rightarrow \mathrm{H}_{\text {fast }}^{0}+\mathrm{H}_{\mathrm{NB}}^{+}, \\
& \mathrm{H}_{\text {bulk }}^{+}+\mathrm{H}_{\mathrm{NB}}^{0} \rightarrow \mathrm{H}_{\text {bulk }}^{0}+\mathrm{H}_{\mathrm{NB}}^{+}, \\
& \mathrm{H}_{\text {fast }}^{+}+\mathrm{H}_{\text {bulk }}^{0} \rightarrow \mathrm{H}_{\text {fast }}^{0}+\mathrm{H}_{\text {bulk }}^{+} .
\end{aligned}
$$

Our interest is in the $\mathrm{H}_{\alpha}$ light created through the charge exchange (CX) between the fast-ions and neutral beam particles (NB-FICX component, Eq. (1)). Simultaneously, the beam particles can also react with bulk ions and create lowenergy neutrals, called halo neutrals, denoted by $\mathrm{H}_{\text {bulk }}^{0}$ in Eq. (2). The $\mathrm{H}_{\alpha}$ light from the halo neutrals can be separated in FICX spectra by selecting a proper wavelength range to observe, since the amount of Doppler shift is different for $\mathrm{H}_{\text {bulk }}^{0}$ and $\mathrm{H}_{\text {fast }}^{0}$. Moreover, the halo neutrals can serve as a source of CX for fast ions, as shown in Eq. (3). The $\mathrm{H}_{\alpha}$ light from this process (halo-FICX component) is difficult to separate from the NB-FICX component, since the velocities of the fast neutrals in Eqs. (1) and (3) are in the same range.

Figure 1 shows an example of FICX spectra for different neutral energies in a simple geometry where the neutrals are assumed to be injected perpendicular to the magnetic field lines and the line of sight (LOS) of the FICXS diagnostic is parallel to the direction of the neutrals. The densities of fast-ions and neutrals are assumed to be the same. If $\mathrm{H}_{\alpha}$ light is emitted from the fast neutrals that are 


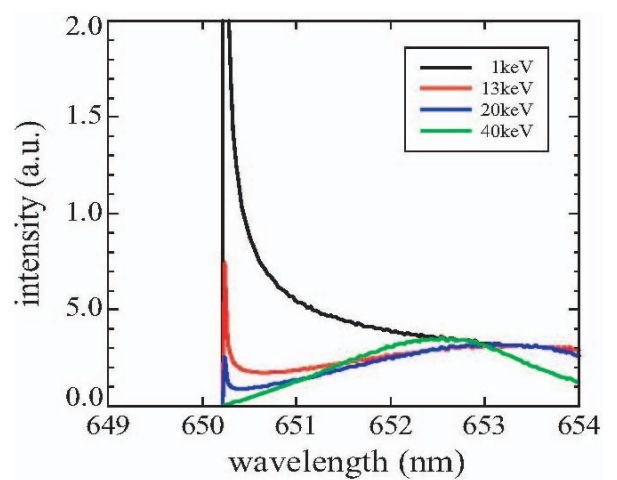

Fig. 1 Examples of Doppler-shifted $\mathrm{H}_{\alpha}$ spectra for different neutral energies $(40,20,13$, and $1 \mathrm{keV})$. Fast-ion energy and pitch angle is fixed at $40 \mathrm{keV}$ and $90^{\circ}$, respectively.

moving parallel to the LOS and toward the observer, their wavelength will be $650.2 \mathrm{~nm}$. Since the fast ions gyrate around the magnetic field lines, the $\mathrm{H}_{\alpha}$ spectra of the FICX process have tail components [4,5]. As shown in the figure, the FICX spectra with cold neutrals $(1 \mathrm{keV})$ have larger intensities than those with NBs. This difference comes from the energy dependence of the CX reaction rate and the change in the relative velocities between the neutrals and fast ions during their gyro-motion. Therefore, it might be necessary to evaluate the effect of halo neutrals on the FICX spectra when blue-shifted $\mathrm{H}_{\alpha}$ emission is measured, as the FICX spectra between perpendicular fast ions and perpendicularly injected NBs.

In this study, the FICX spectra of the halo neutrals are evaluated and their contributions to the FICXS measurements are investigated. Section 2 describes the instrumentation for the FICXS diagnostic in the LHD. In Section 3, typical experimental data are shown and compared to calculated FICX spectra based on the fast ion distributions from a GNET simulation [8] and an expected NB attenuation profile. The halo-FICX components are empirically investigated in this section. The halo effect is also evaluated by using the results of the EIRENE-3D Monte Carlo code [9], as discussed in Section 4. The conclusion is presented in Section 5.

\section{Experimental Setup}

The FICXS diagnostic arrangement in the LHD is shown in Fig. 2. The radial distribution of Doppler-shifted $\mathrm{H}_{\alpha}$ emissions is measured using this arrangement. A radial NB $(40 \mathrm{keV})$ is used as a neutral source for the FICXS measurement in the LHD. The background is subtracted from the foreground measurement and is measured on an identical poloidal cross section at a different toroidal location where no NBs are injected, as shown in Fig. 2. In this study, only the blue-shift components are used because the red-shift components are contaminated by beam emission spectra (BES) of the NB itself. The Doppler-shifted $\mathrm{H}_{\alpha}$ emission is reflected at the lower curved mirrors, which (a)

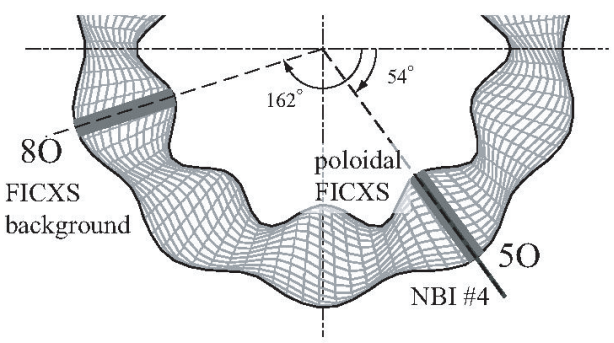

(b)

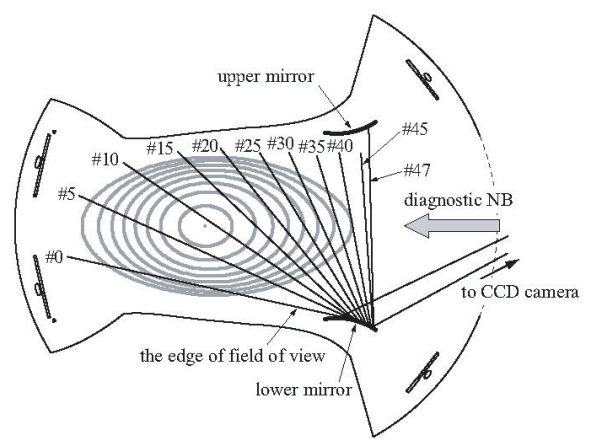

Fig. 2 Schematic drawing of the FICXS diagnostic arrangement on (a) the midplane and (b) the poloidal cross-sectional view of the LHD. Index number is shown besides each LOS. Elliptical gray curves are magnetic flux surfaces of the configuration with a magnetic axis $R_{\mathrm{ax}}=3.6 \mathrm{~m}$ in vacuum.

are located at the outboard side of the vacuum vessel, and are connected to a charged-coupled device (CCD) camera through optical fibers, as shown in Fig. 2. The integration time of the measurement is $150 \mathrm{~ms}$ and the sampling interval is $300 \mathrm{~ms}$.

\section{Empirical Estimation of the Halo Neutral Effect Using Experimental Results}

Figure 3 shows a typical FICX spectrum observed on LOS \#22 for discharge \#82644 at $t=1.3 \mathrm{~s}$, where the magnetic axis position of the vacuum field is $R_{\mathrm{ax}}=3.6 \mathrm{~m}$ and the toroidal field strength at the axis is $B_{\mathrm{t}}=2.5 \mathrm{~T}$. The line-averaged electron density is about $1 \times 10^{19} \mathrm{~m}^{-3}$ and the central electron temperature in the discharge is about $3 \mathrm{keV}$. The FICX spectra along the LOS are reconstructed using the fast-ion energy spectra calculated by the GNET code [8] and the intensity profile of attenuated NBs are calculated by the NBATTEN code [2] using the ADAS database [10]. The reconstructed spectra are also shown in Fig. 3. The calculated spectra using the beam neutrals do not show the hump between $650.4 \mathrm{~nm}$ and $652.2 \mathrm{~nm}$ that appears in the observed spectrum. Neglecting the transport effect of neutrals, the distribution of the halo neutrals can be evaluated from the particle balance equation and is expressed using the density of neutral beam as

$$
n_{\text {halo }}(\vec{r})=n_{\mathrm{NB}}(\vec{r}) \sum_{\mathrm{j}=1}^{3} \frac{<\sigma_{\mathrm{cx}} v>_{\mathrm{ij}} f_{\mathrm{NB}, \mathrm{j}} \gamma_{\mathrm{NB}, \mathrm{j}}}{\left\langle\sigma_{\mathrm{ei}} v>_{\mathrm{ei}}+\left\langle\sigma_{\mathrm{ii}} v>_{\mathrm{ii}}\right.\right.}
$$




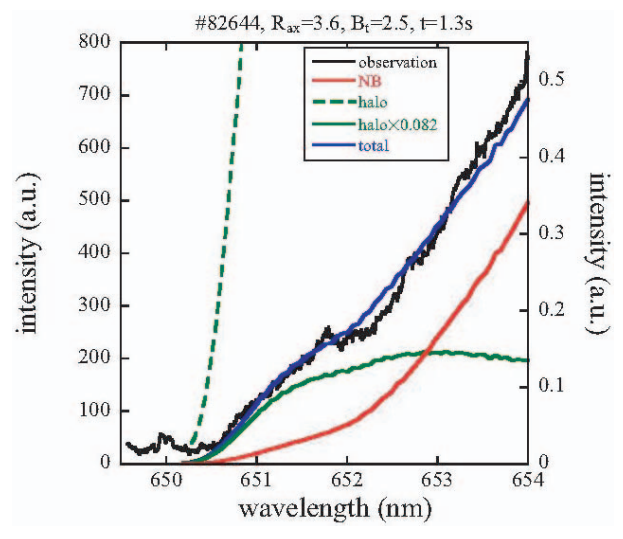

Fig. 3 Comparison of the observed (black) and reconstructed (red) spectra from the GNET and NBATTEN codes. Dashed green line indicates the reconstructed FICX spectra using the results of GNET and the halo neutral distribution evaluated by Eq. (4). Solid green curves indicate the FICX spectra with halo neutrals reduced by a factor of 0.082 . Blue curves indicate the sum of the red and solid green curves.

Here, $\mathrm{a}_{\mathrm{cx}}, \mathrm{a}_{\mathrm{ei}}$, and $\mathrm{a}_{\mathrm{ii}}$ are the cross sections of hydrogen neutrals in the CX reaction with hydrogen ions, in the electron impact ionization, and in the ion impact ionization, respectively. The \langle\rangle$_{\mathrm{ei}}$ and $\left\langle>_{\text {ii }}\right.$ notations indicate the integration over the Maxwellian distribution functions, and $<>_{\mathrm{ij}}$ indicates the integration over the Maxwellian and beam distribution function. Index $\mathrm{j}$ indicates the $\mathrm{j}$-th energy component and $f_{\mathrm{NB}, \mathrm{j}}$ indicates its fraction of the total NB density at the exit port of the NB injector. $Y_{\mathrm{Nb}, \mathrm{j}}$ is the attenuation factor of the NB by the LHD plasmas. Using the neutral density profile calculated by this formula, the halo-FICX spectra can be evaluated. Since Eq. (4) neglects neutral transport, the evaluated neutral density apparently over-estimates the actual value. Thus, an empirical factor for the halo neutral density must be introduced for comparing the halo-FICX spectra to the observed spectra. This factor is chosen to reduce the estimated amount of the halo neutrals on the LOS, so that the calculated FICX spectra reflect experimental observations well. In Fig. 3, the haloFICX spectra are indicated by green lines, and the total FICX spectra, which are the sum of the halo-FICX spectra and the NB-FICX spectra, are indicated in blue. In the figure, an empirical factor of 0.082 is applied. The hump between $650.4 \mathrm{~nm}$ and $652.2 \mathrm{~nm}$ in the observed spectra seems to come from the halo caused by the FICX process. This indicates the importance of evaluating the halo neutral contribution in FICXS measurements.

\section{Evaluation of the Halo Neutrals by the EIRENE Code}

Since the empirical evaluation of the halo neutral contribution is rather ad hoc, it is better to estimate its contribution independently. For this purpose, the EIRENE-

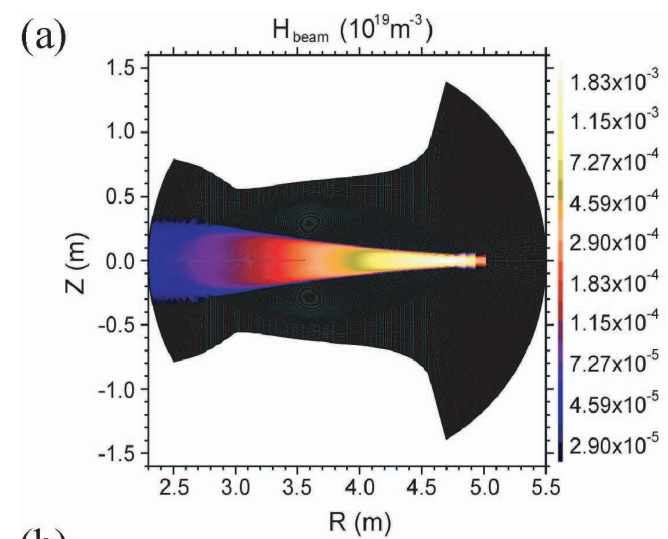

(b)

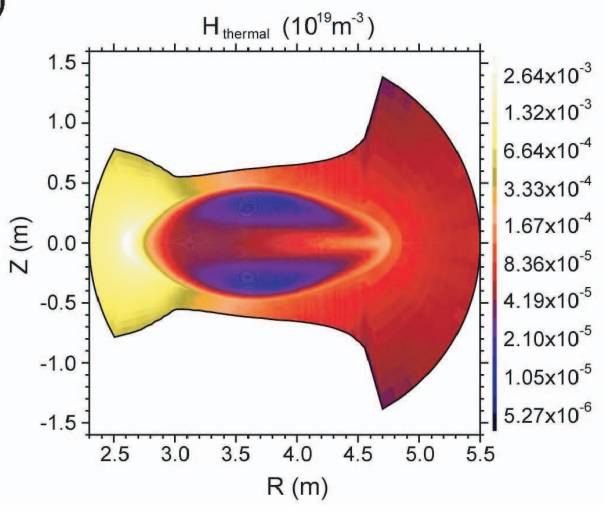

Fig. 4 Spatial density profile of (a) the injected NB and (b) cold neutrals in discharge \#82644 obtained by EIRENE-3D code; divertor recycling is not considered in the calculation.

3D Monte Carlo neutral transport code was applied and a new function for treating the NB injection was developed in the EIRENE-3D code. Figure 4 shows the results of the calculation at the poloidal cross section, where the radial NB was injected and the FICXS LOSs were located. Figure 4 (a) shows the density of the radial beam. The beam was injected from right to left and was focused at the peripheral region of $R \sim 5 \mathrm{~m}$ on the midplane in the figure. The injected beam spreads and attenuates toward the inner wall. The density of the injected NB decreases by order two. The population of cold neutrals in the energy range of the bulk ion temperature is shown in Fig. 4(b). In the calculation, the recycling of hydrogen atoms at the divertor plates is not considered. However, the generation of the cold neutrals by the NB shine-through at the counter-wall of the NB injection port is considered. The cold neutrals that cover the plasma periphery correspond to these neutrals. Note that other cold-neutral components exist around the NB injection path in the core region of the plasma. These are the halo neutrals that we examine in this study. The FICX spectra are calculated by using the neutral distribution obtained by the EIRENE code and the fast-ion spectra obtained by the GNET code, as shown in Fig. 5. The agreement of the estimated and observed spectra is extremely good. 


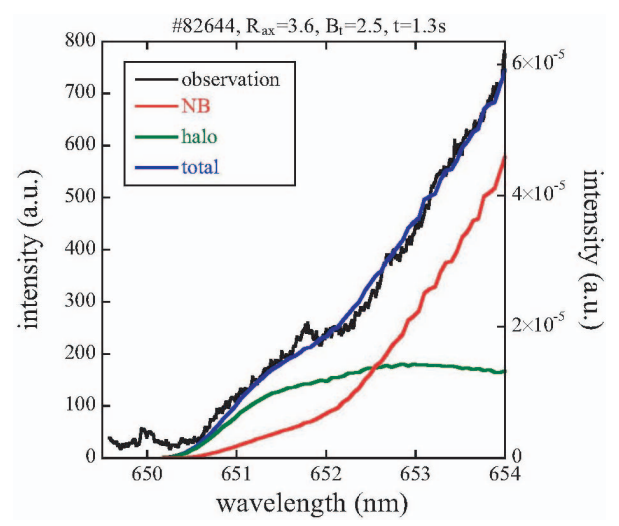

Fig. 5 Comparison between the observed and calculated spectra based on the results of the EIRENE code. Black, red, and green curves indicate experimental observations, the calculated spectrum with an injected $\mathrm{NB}$, and that with halo neutrals, respectively. Blue curve indicates the sum of the calculated spectra.

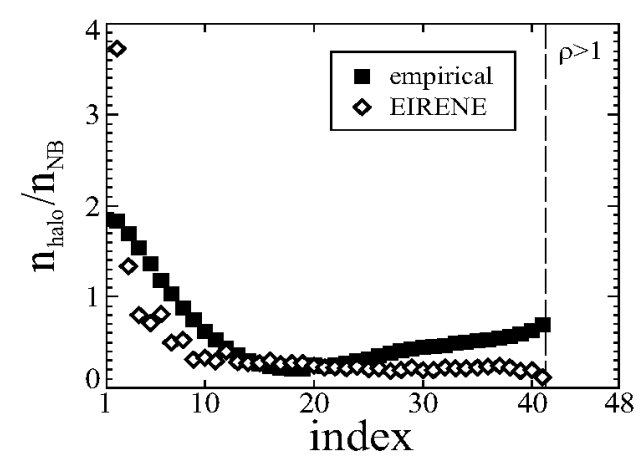

Fig. 6 Distributions of the ratios of $n_{\text {halo }}$ to $n_{\mathrm{NB}}$ for empirical estimations from experimental observations (solid squares) and the results of the EIRENE-3D code (open diamonds).

By using the empirical factor for the halo neutrals discussed in section 3 , the ratios of the halo neutrals to the NB particles on the FICXS LOSs were evaluated and compared to the results of the EIRENE code. Figure 6 shows the spatial distributions of $n_{\text {halo }} / n_{\mathrm{NB}}$ ratios obtained by the empirical method and the EIRENE code. In integrating the ratios on the LOSs, only the region within the last closed flux surface is considered, since few fast ions exist outside this region and the FICX emission from the outer region can be neglected. These show almost the same tendency, but a small difference appears near the edge of the LOS indices. The discrepancy at the outboard side might be due to the modeling of NBs in the EIRENE code. In the code modeling, NBs are assumed to focus on the $R=5 \mathrm{~m}$ point on the midplane with a zero divergent angle, as shown in
Fig. 4 (b). This assumption produces a highly-peaked halo neutral distribution around $Z=0$ at the outboard side of the plasma. Assuming that neutral transport is dominated by diffusion, this peaked profile will provide a higher diffusion velocity than the experimental value and might result in a lower $n_{\text {halo }} / n_{\mathrm{NB}}$ ratio. On the other hand, we have not reached a conclusive description with regards to the discrepancy at the inboard side. Explaining this difference more clearly is a future task.

\section{Conclusion}

The effect of the halo neutrals on the FICXS measurement was investigated in this study. The contributions of the halo-FICX components are evaluated both empirically and by also using the halo neutral distribution calculated by the EIRENE-3D Monte Carlo code. These evaluations confirmed that the hump in the observed spectra between $650.4 \mathrm{~nm}$ and $652.2 \mathrm{~nm}$ was due to the contribution of the halo-FICX process. It was also found that the halo-FICX spectra could exceed the NB-FICX spectra in this wavelength range when the FICX spectra of perpendicular fast ions are measured using radially injected NBs. Thus, the contribution of halo neutrals must be taken into account with this observation geometry, although the direct emission of $\mathrm{H}_{\alpha}$ light from the halo neutrals can be distinguished in the FICX spectra. The spatial profiles of the ratio of the halo neutrals to the injected NBs were also investigated both empirically and by using the EIRENE-3D Monte Carlo code. The two distributions agreed well.

\section{Acknowledgments}

This work was supported by the LHD project budget (NIFS09ULBB512, NIFS09KLBB301, NIFS09ULBB510, and NIFS09KLBB301). This work was also partly supported by the Japan Society for the Promotion of Science (KAKENHI, Nos. 18340189 and 21360457).

[1] M. Osakabe et al., Nucl. Fusion 46, S911 (2006).

[2] M. Osakabe et.al., to be published in Fusion Sci. Technol.

[3] M. Isobe et.al., to be published in Fusion Sci. Technol.

[4] M. Osakabe et al., Rev. Sci. Instrum. 79, 10E519 (2008).

[5] W. W. Heidbrink et al., Plasma Phys. Control. Fusion 46, 1855 (2004).

[6] Y. Luo et al., Rev. Sci. Instrum. 78, 033505 (2007).

[7] M. A. Van Zeeland et al., Plasma Phys. Control. Fusion 51, 055001 (2009).

[8] S. Murakami et al., Fusion Sci. Technol. 46, 241 (2004).

[9] D. Reiter et al., Fusion Sci. Technol. 47, 172 (2005).

[10] H. P. Summers, The ADAS User Manual, version 2.6, 2004. 